\title{
Treatment of adult Burkitt lymphoma with the CALGB 1002 protocol: a single center experience in Jordan
}

\author{
Mohammad Ma'koseh, Rula Amarin, Faris Tamimi, Baha' Sharaf, Alaa Abufara, Omar Shahin, \\ Mohammed K. M. Manassra, Khalid Halahleh \\ Department of Medical Oncology, King Hussein Cancer Center, Amman, Jordan
}

p-ISSN 2287-979X / e-ISSN 2288-0011 https://doi.org/10.5045/br.2021.2021116 Blood Res 2021;56:279-284.

Received on June 14, 2021

Revised on August 28, 2021

Accepted on October 13, 2021

\section{Correspondence to}

Mohammad Ma'koseh, M.D.

Department of Medical Oncology, King

Hussein Cancer Center, Queen Rania

Al-Abdulla Street, Amman 11941, Jordan

E-mail: maakoseh727@yahoo.com

(C) 2021 Korean Society of Hematology

\begin{abstract}
Background
The treatment of adult Burkitt lymphoma with pediatric-based chemotherapy protocols usually results in high cure rates, although with significant toxicity. We report our experience with the Cancer and Leukemia Group B1002 (CALGB 1002) protocol.

\section{Methods}

The files of adult patients diagnosed with Burkitt lymphoma and treated with the CALGB 1002 protocol at King Hussein Cancer Center between 2008 and 2017 were reviewed. Baseline demographics, clinical laboratory features, treatment details, and responses were collected. The correlations between clinical and laboratory variables with event-free survival (EFS) and overall survival (OS) were determined by univariate and multivariate analyses using backward stepwise Cox regression models. EFS and OS were plotted using Kaplan-Meier curves.

Results

This study included 19 patients with a median age of 33 years (range, 19-65). Eleven (58\%) and two (10.5\%) patients had advanced-stage and central nervous system disease, respectively. Among 106 administered cycles, the median interval between cycles was 23 days (range, 19-84 days). Sixteen patients (84\%) achieved a complete response. After a median follow-up of 40.8 months, the 3-year EFS and OS rates were $78.95 \%$. Patients with a low-risk International Prognostic Index (IPI) had better survival than those with intermediate-or high-risk IPI. Grade III-IV hematological toxicities occurred in $88 \%$ of patients, while $73 \%$ had grade III-IV mucositis.
\end{abstract}

\section{Conclusion}

In adult Burkitt lymphoma, the CALGB 1002 protocol provides high cure rates and can be administered promptly, but is associated with significant toxicity. Risk-adapted approaches and other, less toxic, chemotherapeutic regimens should be considered.

Key Words Burkitt lymphoma, CALGB 1002, International Prognostic Index

\section{INTRODUCTION}

Burkitt lymphoma (BL) is a highly aggressive B-cell non-Hodgkin lymphoma (NHL) characterized by a high proliferation rate (Ki 67 of approximately 100\%) and deregulation of the MYC gene. It is more common in the pediatric age group, peaking around 10 years of age, but accounts for only about $1 \%$ of all NHL in adults [1-3].

Clinically, BL often presents with rapidly enlarging masses and extranodal site involvement, especially the bone marrow and central nervous system (CNS). Owing to its rapid growth, early diagnosis and prompt treatment initiation are important [4].

The treatment of $\mathrm{BL}$ in adults is challenging because of the lack of randomized trials to define the best initial regimen. Most first-line regimens are adopted from intensive pediatric protocols and include a combination of anthracycline, alkylator, vincristine, etoposide, and agents to treat and prevent CNS disease [5].

Cancer and Leukemia Group B (CALGB) developed an intensive regimen (CALGB 9251) that initially included mul- 
ti-agent chemotherapy with prophylactic cranial radiotherapy and 12 doses of intrathecal chemotherapy (ITC) [6]. As $61 \%$ of patients in the first cohort developed severe neurological toxicity, in the second cohort, prophylactic cranial radiotherapy was administered only to patients with bone marrow involvement, and only seven ITC doses were administered. The 5-year overall survival (OS) rate was 52\%. In a follow-up study (CALGB 1002), the addition of rituximab resulted in improvements of 4-year event-free survival (EFS) and $O S$ to $78 \%$ and $74 \%$, respectively [7].

The CALGB 1002 protocol was used for the treatment of most BL cases in our center. Our study aimed to describe the characteristics and outcomes of adult BL patients treated with the CALGB 1002 protocol.

\section{MATERIALS AND METHODS}

We retrospectively analyzed the medical records of adult patients diagnosed with BL and treated with the CALGB 1002 regimen at the Medical Oncology Department, King Hussein Cancer Center in Jordan between January 2008 and December 2017.

The following variables were retrieved from patient charts and electronic medical records: age, sex, Eastern Cooperative Oncology Group (ECOG) performance status, initial lactate

Table 1. Cancer and Leukemia Group B1002 (CALGB 1002) protocol.

\begin{tabular}{|c|c|c|}
\hline \multicolumn{3}{|c|}{ Cycle 1} \\
\hline Drug & Dose & Days \\
\hline Cyclophosphamide & $200 \mathrm{mg} / \mathrm{m}^{2}$ & $1-5$ \\
\hline Prednisolone & $60 \mathrm{mg} / \mathrm{m}^{2}$ & $1-7$ \\
\hline \multicolumn{3}{|c|}{ Cycles 2, 4, and 6} \\
\hline Drug & Dose & Days \\
\hline Ifosfamide & $800 \mathrm{mg} / \mathrm{m}^{2}$ & $1-5$ \\
\hline Mesna & $200 \mathrm{mg} / \mathrm{m}^{2}$ & 1 to 5 (At h 0,4 , and 8 after ifosfamide) \\
\hline Methotrexate & $1,500 \mathrm{mg} / \mathrm{m}^{2}$ & 1 \\
\hline Leucovorin & \multicolumn{2}{|c|}{$\begin{array}{l}25 \mathrm{mg} / \mathrm{m}^{2} 36 \mathrm{~h} \text { after starting methotrexate infusion, } \\
\text { then } 10 \mathrm{mg} / \mathrm{m}^{2} \text { until methotrexate level }<0.05 \mu \mathrm{mol} / \mathrm{L}\end{array}$} \\
\hline Vincristine & $2 \mathrm{mg}$ & 1 \\
\hline Cytarabine & $1,000 \mathrm{mg} / \mathrm{m}^{2}$ & $4-5$ \\
\hline Etoposide & $80 \mathrm{mg} / \mathrm{m}^{2}$ & $4-5$ \\
\hline \multicolumn{3}{|l|}{ Intrathecal } \\
\hline Methotrexate & $15 \mathrm{mg}$ & 1 \\
\hline Cytarabine & $40 \mathrm{mg}$ & \\
\hline Hydrocortisone & $50 \mathrm{mg}$ & \\
\hline \multirow[t]{5}{*}{ Rituximab } & Cycle 2: & \\
\hline & $50 \mathrm{mg} / \mathrm{m}^{2}$, then & 8 \\
\hline & $375 \mathrm{mg} / \mathrm{m}^{2}$ & 10 \\
\hline & Cycles 4 and 6: & \\
\hline & $375 \mathrm{mg} / \mathrm{m}^{2}$ & 8 \\
\hline Filgrastim & $5 \mathrm{mcg} / \mathrm{kg}$ & Starting on day 7 \\
\hline \multicolumn{3}{|c|}{ Cycles 3, 5 and 7} \\
\hline Drug & Dose & Days \\
\hline Cyclophosphamide & $200 \mathrm{mg} / \mathrm{m}^{2}$ & $1-5$ \\
\hline Methotrexate & $1,500 \mathrm{mg} / \mathrm{m}^{2}$ & 1 \\
\hline Leucovorin & \multicolumn{2}{|c|}{$\begin{array}{c}25 \mathrm{mg} / \mathrm{m}^{2} 36 \mathrm{~h} \text { after starting methotrexate infusion, then } \\
10 \mathrm{mg} / \mathrm{m}^{2} \text { until methotrexate level }<0.05 \mu \mathrm{mol} / \mathrm{L}\end{array}$} \\
\hline Vincristine & $2 \mathrm{mg}$ & 1 \\
\hline Doxorubicin & $25 \mathrm{mg} / \mathrm{m}^{2}$ & $4-5$ \\
\hline Dexamethasone & $10 \mathrm{mg} / \mathrm{m}^{2}$ & $4-5$ \\
\hline \multicolumn{3}{|l|}{ Intrathecal } \\
\hline Methotrexate & $15 \mathrm{mg}$ & 1 \\
\hline Cytarabine & $40 \mathrm{mg}$ & \\
\hline Hydrocortisone & $50 \mathrm{mg}$ & \\
\hline Rituximab & $375 \mathrm{mg} / \mathrm{m}^{2}$ & 8 \\
\hline Filgrastim & $5 \mathrm{mcg} / \mathrm{kg}$ & Starting on day 7 \\
\hline
\end{tabular}


dehydrogenase (LDH) level, stage on time of diagnosis, and extra-nodal sites involved. In addition, treatment details including the timing of chemotherapy administration, ITC, hematological and non-hematological toxicities, response, and relapse were also obtained.

The details of the treatment protocol are provided in Table 1.

Staging was performed according to the Lugano staging system based on computed tomography (CT) scans [8]. Bulky disease was defined as tumor bulk $>10 \mathrm{~cm}$ in the transverse or coronal diameters. The International Prognostic Index (IPI) was calculated as initially described using age, performance status, LDH level, stage, and number of extranodal sites [9]. The patients were considered to have low risk if they met all of the following criteria: Lugano stage I-II, normal LDH, ECOG performance status of $0-1$, and tumor mass $<10 \mathrm{~cm}$ [10]. Patients who did not fulfill the criteria for low-risk disease were considered to have high-risk disease.

Response evaluation was performed based on the modified Cheson lymphoma response criteria [11]. Toxicity grading was done using Common Terminology Criteria for Adverse Events (CTCAE) version 5.0 [12].

EFS was calculated from the time of diagnosis until the

Table 2. Patient characteristics.

\begin{tabular}{lc}
\hline \multicolumn{1}{c}{ Variable $(\mathrm{N}=19)$} & $\mathrm{N}(\%)$ \\
\hline Age & Median: $33(19-65)$ \\
Age $>60 \mathrm{yr}$ & $1(5.3)$ \\
Male & $12(63)$ \\
Stage & \\
I-II & $8(42.1)$ \\
III-IV & $11(58)$ \\
ECOG performance status & \\
$0-1$ & $15(78.9)$ \\
2 or more & $4(21.1)$ \\
High LDH & $10(52.6)$ \\
Extranodal involvement & $14(73.6)$ \\
Number of extranodal sites & \\
1 & $7(36.8)$ \\
$>1$ & $7(36.8)$ \\
Bone marrow involvement & $2(10.5)$ \\
CNS involvement & $2(10.5)$ \\
Bulky disease & $5(26.3)$ \\
B symptoms & $5(26.3)$ \\
Risk classification & \\
Low & $6(31.5)$ \\
High & $13(68.5)$ \\
International Prognostic Index & \\
Low (0-1) & $9(47.3)$ \\
Low-intermediate $(2)$ & $4(21.1)$ \\
High-intermediate (3) & $3(15.8)$ \\
High (4-5) & $3(15.8)$ \\
\hline
\end{tabular}

B symptoms: fever $\left(>38^{\circ} \mathrm{C}\right)$, weight loss $(>10 \%$ over $6 \mathrm{mo})$, and drenching night sweats.

Abbreviations: CNS, central nervous system; LDH, lactate dehydrogenase. time of death, progression, or relapse, while OS was calculated from the time of diagnosis until death from any cause or the date of the last follow-up.

The correlations of different clinical and laboratory variables with EFS and OS were determined by univariate and multivariate analyses using the backward stepwise Cox regression model. The EFS and OS were calculated and plotted using the Kaplan-Meier method.

\section{RESULTS}

\section{Patient characteristics}

This study included 19 patients, 12 (63\%) of whom were male. The median age was 33 years (range, 19-65 yr). Eleven patients $(58 \%)$ had advanced-stage disease (stage III or IV), and $14(73.6 \%)$ had extranodal involvement. CNS involvement was found in two patients $(10.5 \%)$. Bone marrow involvement was also found in two patients (10.5\%), and 13 patients $(68 \%)$ had high-risk disease. The detailed patient characteristics are shown in Table 2.

\section{Treatment and response}

The median time from diagnosis to treatment initiation was 11 days (range, 8-56 days). A total of 106 cycles were administered, with the median interval between cycles of 23 days (range, 19-84 days). All patients were administered a median of six doses of ITC (range, 2-6). Three patients $(15.8 \%)$ received craniospinal radiotherapy.

Sixteen patients $(84 \%)$ achieved a complete response (CR), while the other three patients failed to achieve a CR (one had a partial response: one died of chemotherapy-related sepsis before having a response evaluation, and one developed disease progression).

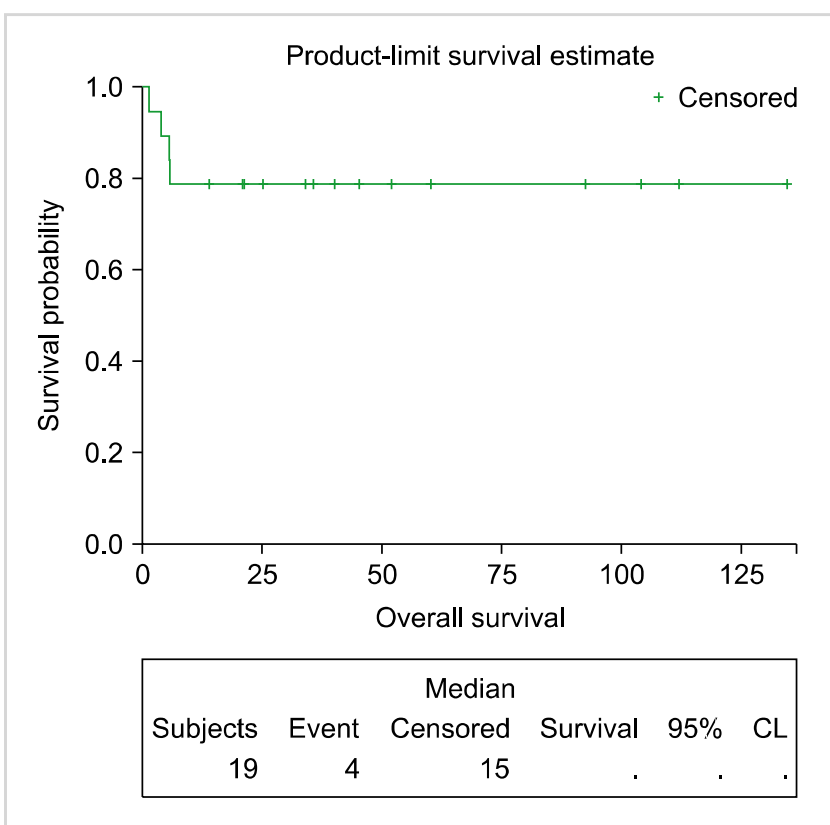

Fig. 1. Overall survival. 
After a median follow-up of 36 months, the 3-year EFS and OS rates were both $78.9 \%$ (Figs. 1, 2).

Four patients $(21.1 \%)$ died: two from disease progression and two from chemotherapy-related complications.

Stratification of the patients according to IPI showed a significant difference in 3-year OS rates between patients with low IPI and those with intermediate or high IPI (100\% vs. $60 \%, P=0.038$ ) (Fig. 3). In contrast, the 3 -year OS rates did not differ significantly between patients with low- and high-risk disease (100\% vs. $69 \%, P=0.147)$ (Fig. 4).

Univariate analysis showed that abnormal LDH, intermediate or high IPI, and bulky disease were correlated with worse OS. However, none of these factors was significant in the multivariate analysis (Table 3).

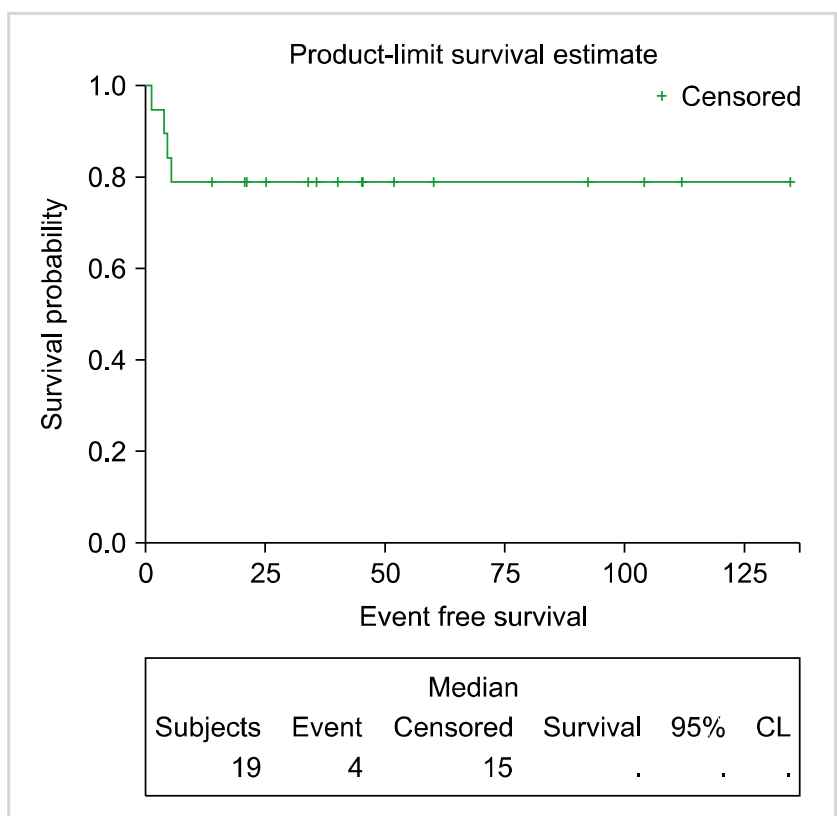

Fig. 2. Event-free survival.

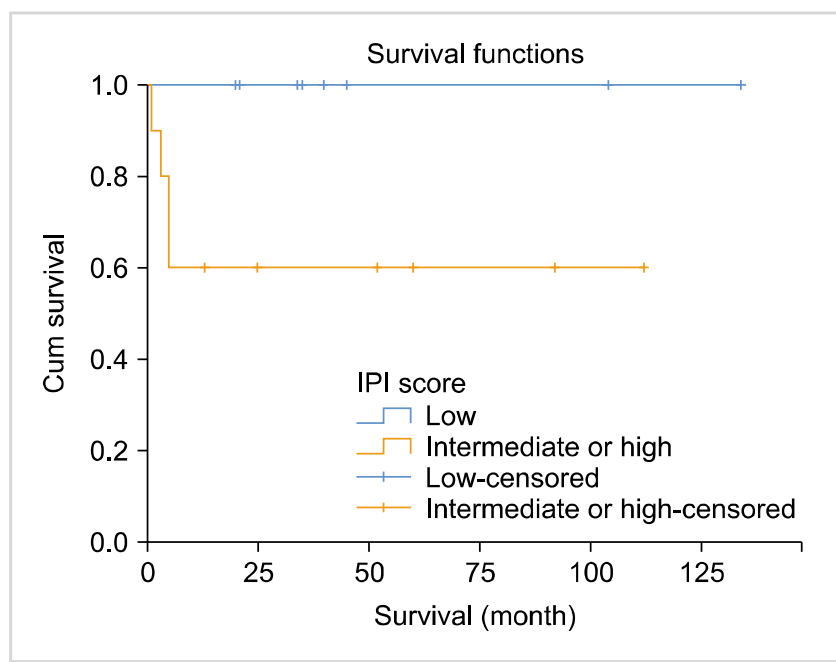

Fig. 3. Overall survival according to low versus high International Prognostic Index (IPI) values $(P=0.038)$.

\section{Toxicity}

Hematological toxicities, including grade III-IV neutropenia and thrombocytopenia, were both found in $88 \%$ of patients, while febrile neutropenia occurred in $89 \%$ of patients. The most important grade III-IV non-hematological toxicities were mucositis, which occurred in 13 patients (72\%). Two patients (10.5\%) from chemotherapy-related toxicities, one after the second cycle and the second after the fifth cycle.

\section{DISCUSSION}

We reported our experience with the treatment of $\mathrm{BL}$ with a uniform chemotherapy protocol. To our knowledge, only one study has previously reported real-life experiences with the CALGB protocol in BL [13]. Comparison to the original study by Rizzieri et al. [7], our patients had more favorable features including younger median age (33 vs. 43 yr) and fewer patients with initial bone marrow involvement ( $10.5 \%$ vs. $28 \%$ ), B symptoms ( $26 \%$ vs. $48 \%$ ), and high-intermediate or high IPI scores (32\% vs. $47 \%)$. However, the EFS and OS rates were almost comparable, which might be explained by the smaller sample size and relatively higher treatment-related mortality $(10.5 \%$ vs. $6.6 \%)$.

Interestingly, as observed in most BL trials, all of our patients who achieved CR after completion of treatment continued to be disease-free until the last follow-up.

Several features of the CALGB 1002 protocol contribute to its high efficacy, including the use of non-cross-resistant chemotherapeutic agents, lack of treatment delays (as demonstrated in our study), and aggressive CNS prophylaxis [14]. However, the major disadvantage of the CALGB 1002 protocol is the lack of a risk-stratified approach. This may result in unnecessary exposure of low-risk patients to toxic treatments. Most trials stratify patients according to their initial features as well as their interim response to treatment.

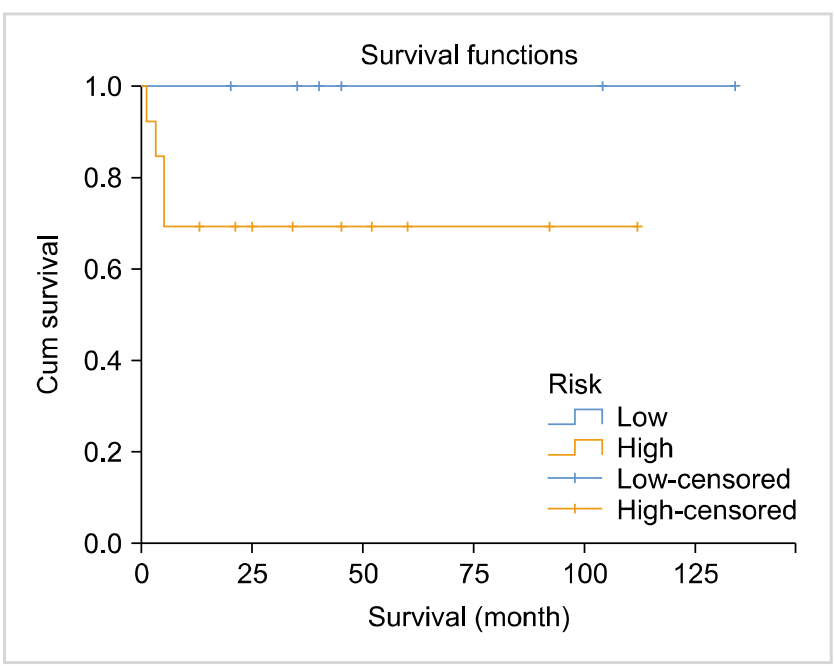

Fig. 4. Overall survival in patients with low- and high-risk disease $(P=0.138)$. 
Table 3. Univariate and multivariate analysis.

\begin{tabular}{|c|c|c|c|}
\hline Variable & Univariate analysis $(P)$ & OR & Multivariate analysis $(P)$ \\
\hline Age at diagnosis $>60 \mathrm{yr}$ & 0.62 & & \\
\hline Ann Arbor stage III-IV & 0.06 & & \\
\hline Serum LDH level $>1 \times$ normal & 0.04 & 70.66 & 0.97 \\
\hline$>1$ extranodal site & 0.55 & & \\
\hline Bone marrow involvement & 0.68 & & \\
\hline CNS involvement & 0.68 & & \\
\hline International Prognostic Index & 0.04 & 70.66 & 0.97 \\
\hline B symptoms & 0.30 & & \\
\hline Bulky disease & 0.01 & 11.37 & 0.49 \\
\hline
\end{tabular}

Abbreviations: CNS, central nervous system; LDH, lactate dehydrogenase; OR, odds ratio.

The features used to define "high risk" included one of the following: elevated LDH, ECOG performance status $\geq 2$, and bulky disease $(7-10 \mathrm{~cm})$. Although using this definition will classify $80-90 \%$ of BL as high risk [15], it was successfully implemented in adopting a risk-stratified treatment for BL. Mead et al. [10] tested this approach in 52 patients treated with the Magrath regimen, CODOX-M/IVAC [cyclophosphamide, doxorubicin, vincristine, methotrexate (CODOX-M) alternating with ifosfamide, cytarabine, and etoposide (IVAC)]. Low-risk patients were administered three cycles of CODOX-M, while high-risk patients were administered four cycles of CODOX-M/IVAC. The 2-year EFS rates were $83 \%$ and $56 \%$ in low-risk and high-risk groups, respectively.

Risk-stratified treatment was also tested in 113 patients treated with a dose-adjusted EPOCH-R (etoposide, prednisone, vincristine, cyclophosphamide, doxorubicin, and rituximab) protocol [16]. The low-risk patients were administered two cycles, and a third cycle if PET-CT scans showed no evidence of disease. Patients who did not achieve complete remission after the second cycle and all high-risk patients were administered six cycles. The 4-year EFS rates were $100 \%$ and $82.1 \%$ in the low- and high-risk groups, respectively.

Similar to the results of many BL studies $[7,17,18]$ our study findings confirmed the significantly inferior outcomes of patients with intermediate and high IPI compared to those of patients with lower IPI scores (3-yr EFS: 60\% vs. 100\%). The best management of $\mathrm{BL}$ with high-intermediate and high IPI remains unclear. A recent phase II UK-NCI trial showed that the use of rituximab with CODOX-M/IVAC was associated with more favorable outcomes [2-yr progression-free survival (PFS) of $77.2 \%$ and OS of $80.7 \%$ ] [19]. In addition, in a multicenter trial that reported the outcomes of patients treated with dose-adjusted EPOCH-R, the outcomes were favorable regardless of IPI [16].

The use of pediatric-inspired protocols in adult $\mathrm{BL}$ is associated with substantial hematological and non-hematological toxicities, with treatment-related mortality occurring in 5$10 \%$ of patients $[7,10,20-22]$. The use of dose-adjusted EPOCH-R was associated with favorable outcomes (4-yr EFS and OS of $84.5 \%$ and $87 \%$, respectively) with much lower toxicities. However, patients with CNS involvement were at high risk for treatment failure, with an EFS of only $40 \%$ [15]. Moreover, a recent multicenter cohort study showed that CNS relapse was more common in dose-adjusted EPOCH-R than in CODOX-MI/VAC and Hyper-CVAD (13\% vs. $4 \%$ and $3 \%$, respectively) [23]. Incorporation of high-dose methotrexate with dose-adjusted EPOCH-R has been successfully attempted $[24,25]$ and may help to improve the outcomes of patients with CNS involvement and decrease the rate of CNS relapse.

Our study has important limitations, including its retrospective nature and the small number of patients observed in most of the BL studies.

In conclusion, despite its substantial toxicities, CALGB 1002 could be administered promptly, resulting in high cure rates among patients with BL. Adoption of risk-adapted approaches, as well as the use of less intensive chemotherapeutic protocols, would direct future progress in the treatment of BL.

\section{Authors' Disclosures of Potential Conflicts of Interest}

No potential conflicts of interest relevant to this article were reported.

\section{REFERENCES}

1. Dunleavy K. Approach to the diagnosis and treatment of adult Burkitt's lymphoma. J Oncol Pract 2018;14:665-71.

2. Morton LM, Wang SS, Devesa SS, Hartge P, Weisenburger DD, Linet MS. Lymphoma incidence patterns by WHO subtype in the United States, 1992-2001. Blood 2006;107:265-76.

3. Kalisz K, Alessandrino F, Beck R, et al. An update on Burkitt lymphoma: a review of pathogenesis and multimodality imaging assessment of disease presentation, treatment response, and recurrence. Insights Imaging 2019;10:56.

4. Kasamon YL, Swinnen LJ. Treatment advances in adult Burkitt lymphoma and leukemia. Curr Opin Oncol 2004;16:429-35.

5. Jacobson C, LaCasce A. How I treat Burkitt lymphoma in adults. 
Blood 2014;124:2913-20.

6. Rizzieri DA, Johnson JL, Niedzwiecki D, et al. Intensive chemotherapy with and without cranial radiation for Burkitt leukemia and lymphoma: final results of Cancer and Leukemia Group B Study 9251. Cancer 2004;100:1438-48.

7. Rizzieri DA, Johnson JL, Byrd JC, et al. Improved efficacy using rituximab and brief duration, high intensity chemotherapy with filgrastim support for Burkitt or aggressive lymphomas: cancer and Leukemia Group B study 10 002. Br J Haematol 2014; 165:102-11.

8. Cheson BD, Fisher RI, Barrington SF, et al. Recommendations for initial evaluation, staging, and response assessment of Hodgkin and non-Hodgkin lymphoma: the Lugano classification. J Clin Oncol 2014;32:3059-68.

9. International Non-Hodgkin's Lymphoma Prognostic Factors Project. A predictive model for aggressive non-Hodgkin's lymphoma. N Engl J Med 1993;329:987-94.

10. Mead GM, Sydes MR, Walewski J, et al. An international evaluation of CODOX-M and CODOX-M alternating with IVAC in adult Burkitt's lymphoma: results of United Kingdom Lymphoma Group LY06 study. Ann Oncol 2002;13:1264-74.

11. Cheson BD, Pfistner B, Juweid ME, et al. Revised response criteria for malignant lymphoma. J Clin Oncol 2007;25:579-86.

12. National Cancer Institute. Division of cancer treatment and diagnosis. Common Terminology Criteria for Adverse Events (CTCAE) v5.0. Bethesda, MD: National Cancer Institute, 2020. (Accessed May 20, 2021, at https://ctep.cancer.gov/protocoldevelopment/ electronic_applications/docs/CTCAE_v5_Quick_Reference_8. $5 x 11 . p d f)$.

13. Lee D, Kim S, Ko OB, et al. Burkitt's lymphoma in Korea: clinical manifestations and efficacy of modified CALGB 9251 regimen (BNHL). Blood (ASH Annual Meeting Abstracts) 2005;106 (Suppl):4661.

14. Blum KA, Lozanski G, Byrd JC. Adult Burkitt leukemia and lymphoma. Blood 2004;104:3009-20.

15. Roschewski M, Dunleavy K, Abramson JS, et al. Multicenter study of risk-adapted therapy with dose-adjusted EPOCH-R in adults with untreated Burkitt lymphoma. J Clin Oncol 2020;38:2519-29.
16. Evens AM, Carson KR, Kolesar J, et al. A multicenter phase II study incorporating high-dose rituximab and liposomal doxorubicin into the CODOX-M/IVAC regimen for untreated Burkitt's lymphoma. Ann Oncol 2013;24:3076-81.

17. Hoelzer D, Walewski J, Döhner H, et al. Improved outcome of adult Burkitt lymphoma/leukemia with rituximab and chemotherapy: report of a large prospective multicenter trial. Blood 2014;124:3870-9.

18. Intermesoli $T$, Rambaldi A, Rossi G, et al. High cure rates in Burkitt lymphoma and leukemia: a Northern Italy Leukemia Group study of the German short intensive rituximab-chemotherapy program. Haematologica 2013;98:1718-25.

19. McMillan AK, Phillips EH, Kirkwood AA, et al. Favourable outcomes for high-risk diffuse large B-cell lymphoma (IPI 3-5) treated with front-line R-CODOX-M/R-IVAC chemotherapy: results of a phase 2 UK NCRI trial. Ann Oncol 2020;31:1251-9.

20. Barnes JA, Lacasce AS, Feng Y, et al. Evaluation of the addition of rituximab to CODOX-M/IVAC for Burkitt's lymphoma: a retrospective analysis. Ann Oncol 2011;22:1859-64.

21. Diviné $M$, Casassus $P$, Koscielny $S$, et al. Burkitt lymphoma in adults: a prospective study of 72 patients treated with an adapted pediatric LMB protocol. Ann Oncol 2005;16:1928-35.

22. Patekar M, Gogia A, Tiwari A, et al. Adult Burkitt lymphoma: an institutional experience with a uniform chemotherapy protocol. South Asian J Cancer 2018;7:195-9.

23. Zayac AS, Evens AM, Danilov A, et al. Outcomes of Burkitt lymphoma with central nervous system involvement: evidence from a large multicenter cohort study. Haematologica 2021;106: 1932-42.

24. Sun P, Wang Y, Chen C, et al. R-split-EPOCH plus high dose methotrexate in untreated diffuse large $\mathrm{B}$ cell lymphoma with MYC rearrangement or double expression of $M Y C$ and $B C L-2$. J Cancer 2021;12:2059-64.

25. Chihara D, Fowler NH, Oki Y, et al. Dose-adjusted EPOCH-R and mid-cycle high dose methotrexate for patients with systemic lymphoma and secondary CNS involvement. Br J Haematol 2017;179:851-4. 